\title{
Stationary hyperboloidal slicings with evolved gauge conditions
}

\author{
Frank Ohme ${ }^{1}$, Mark Hannam ${ }^{2}$, Sascha Husa ${ }^{3}$ and Niall Ó Murchadha ${ }^{2}$ \\ ${ }^{1}$ Max-Planck-Institut für Gravitationsphysik, Albert-Einstein-Institut, Am Mühlenberg 1, \\ 14476 Golm, Germany \\ 2 Physics Department, University College Cork, Cork, Republic of Ireland \\ ${ }^{3}$ Departament de Física, Universitat de les Illes Balears, Cra. Valldemossa Km. 7.5, \\ Palma de Mallorca E-07122, Spain \\ E-mail: frank.ohme@aei.mpg.de
}

Received 13 May 2009, in final form 27 July 2009

Published 18 August 2009

Online at stacks.iop.org/CQG/26/175014

\begin{abstract}
We analyze stationary slicings of the Schwarzschild spacetime defined by members of the Bona-Massó family of slicing conditions. Our main focus is on the influence of a non-vanishing offset to the trace of the extrinsic curvature, which forbids the existence of standard Cauchy foliations but at the same time allows gauge choices that are adapted to include null infinity $(\mathscr{I})$ in the evolution. These hyperboloidal slicings are especially interesting for observing outgoing gravitational waves. We show that the standard $1+\log$ slicing condition admits no overall regular hyperboloidal slicing, but by appropriately combining with harmonic slicing, we construct a gauge condition that leads to a strongly singularity-avoiding hyperboloidal foliation that connects the black hole to $\mathscr{I}$.
\end{abstract}

PACS numbers: 04.20.Ex, 04.25.Dm, 04.30.Db, 95.30.Sf

(Some figures in this article are in colour only in the electronic version)

\section{Introduction}

The principal motivation driving the development of numerical relativity has been to treat astrophysically relevant situations in full general relativity and make predictions for physical measurements - in particular for future observations of gravitational waves (GWs). For many years chronic instabilities in numerical codes for simulating the coalescence of black-hole binaries posed a major obstacle for the field, and correspondingly the codes currently used for black-hole simulations originate from a time when the main focus of the field was on the stability of evolution codes, rather than the accuracy of the GW content of the numerically generated spacetimes. Subsequent developments have led to the production of waveforms 
that are accurate enough for many applications in GW astronomy in the near future [1], but succeeding generations of detectors (e.g. LISA [2, 3] or the Einstein telescope [4]) will however significantly increase the accuracy requirements for numerical simulations.

Following the initial breakthrough of 2005 [5-7], a wealth of information on the coalescence of black holes has already been learned from numerical simulations (see [8, 9] for overviews), and the accuracy of numerical codes has increased dramatically (see e.g. [1]). If we are to extract the maximum possible physical and astrophysical information from GW observations, however, we will eventually require yet more accurate, and unambiguous, calculations of the GW signals from these systems. The calculation of the GW signal is complicated in general relativity because observers of signals at astronomical distances from the sources are appropriately idealized by quantities defined at null infinity [10-12], i.e., locations arbitrarily far from the source along lightlike directions. In most numerical codes one calculates an approximation to the GW signal at some set of finite distances (on the order of $\sim 100 M$, where $M$ is the total mass of the system) from the source, and extrapolates the result to null infinity the same way one extrapolates the results from runs at different spacings of the numerical grid to vanishing grid spacing. This procedure is cumbersome, computationally expensive, and, often more importantly, prone to numerical errors and oddities of the numerical coordinates that dominate the error estimate of the final signal.

A preferable approach would be to include null infinity in the numerical code, and there has indeed been much recent progress in this direction, for example in characteristic evolution [13-15], and the hyperboloidal initial-value problem [16, 17]. In this paper we are concerned with the latter approach, namely constructing asymptotically null slices of a black-hole spacetime, with a view to adapting our methods in the future to the construction of hyperboloidal multiple-black-hole initial data for use in numerical simulations. The construction of hyperboloidal initial data is however related to the method that will be used to evolve them, and in particular to the gauge conditions, which will be the focus of this paper.

The idea of evolving the Einstein equations as a Cauchy problem along a foliation of spacelike surfaces that reach null infinity was pioneered by Friedrich [18, 19] (see [20-23] for early overviews on numerical work). It was recognized early on that including null infinity in a numerical simulation naturally involves a compactification of an infinite physical domain onto a finite grid, and is prone to lead to singularities in the differential equations that we wish to solve (for a suggestion to use more generalized slices see [24]). Finding a convenient regularization of these equations is one of the major obstacles to the use of null infinity in numerical simulations. A point of view that has been advocated in recent years [16, 25-27] is that before formulating the concrete system of equations to be solved, the geometric structure of null infinity, which expresses the asymptotically Minkowskian nature of radiation spacetimes, should be made as manifest as possible in the coordinate gauge conditions that will be used. Such a strategy has worked very well for characteristic evolution [15, 28]. The hope is that this will simplify the regularization problem, keep the resulting equations as simple as possible, and carry over many techniques from 'conventional' numerical-relativity simulations on finite Cauchy slices.

Before doing this, one must settle on gauge conditions. It is a common practice in numerical relativity, and ubiquitous in current black-hole-binary codes, to evolve gauge conditions with hyperbolic equations. For example, the popular moving-puncture method employs gauge conditions chosen from the Bona-Massó family [29] (see however [30-32] for elliptic-hyperbolic approaches).

Although one ultimately wants to simulate a dynamical spacetime, stationary solutions play an important role: in many applications perturbations ultimately radiate away, and a 
stationary solution is approached. This is true in particular for astrophysical black-hole spacetimes. It is therefore fruitful to first think in terms of stationary solutions with respect to the gauge conditions. In fact, it is somewhat surprising that the search for stationary representations of black holes which are compatible with the gauge conditions one is using was not posed much earlier as one of the fundamental questions in numerical relativity. Stationary solutions also motivate more suitable numerical methods and forms of initial data. The importance of time-independent descriptions of Minkowski spacetime as a starting point for numerical approaches to the hyperboloidal initial value problem and as a test case for the conformal field equations approach has been discussed in [23]. For recent work on the associated problem of freezing the coordinate position of null infinity in compactified evolutions see [33, 34].

In [35-37] we have discussed explicit stationary representations describing a nonspinning black hole, which are consistent with the specific gauge conditions used in the moving-puncture method and represent a trumpet geometry: the slice extends from a throat at some finite value $R_{0}$ of the Schwarzschild radial coordinate out to spatial infinity. In [34] such maximally sliced 'trumpet data' have been matched explicitly to a family of stationary hyperboloidal slices. In this paper, we construct such 'hyperboloidal trumpet' slices directly from an evolved gauge condition, with the hope that it will provide a useful starting point for developing a method for the simulation of black-hole-binary spacetimes with asymptotically null slices. We first study the compatibility of the Bona-Massó family of slicing conditions [29] with regular stationary hyperboloidal slices of Schwarzschild-Kruskal spacetime. We find incompatibility for the popular ' $1+\log$ ' subfamily, but compatibility for harmonic slicing and certain 'hybrid' conditions, which we construct to yield stationary slices which are both hyperboloidal and singularity avoiding, and which are therefore interesting regarding the generalization of the moving-puncture method to the hyperboloidal problem.

\section{Preliminaries}

\subsection{Geometry of hyperboloidal slices}

Before embarking on technicalities, let us note that a crucial geometric quantity in our work is the (trace of the) extrinsic curvature-in this paper we will choose the convention that the extrinsic curvature of a three-dimensional spacelike hypersurface is defined as the Lie derivative with respect to the timelike unit normal $n^{a}$ of the induced metric $h_{a b}, K_{a b}=+\frac{1}{2} \mathcal{L}_{n} h_{a b}$, with a positive sign. This sign convention is unfortunately not common in numerical relativity, but we believe it is beneficial for physical intuition: positive mean curvature $K=h^{a b} K_{a b}$ then signifies an expanding volume element, while negative $K$ signifies contraction.

While we are interested in the asymptotic structure of slices, in order to simplify notation we will actually not directly work with the conformal compactification picture [10], which has become a standard tool [38], but we nevertheless want to define hyperboloidal slices as regular spacelike hypersurfaces in a compactified spacetime in the sense of Penrose [10]. It then follows for the physical trace of the extrinsic curvature $K$, that $K>0$ at future null infinity $\mathscr{I}^{+}$, and $K<0$ at past null infinity $\mathscr{I}^{-}$. The name 'hyperboloidal' stems from the fact that such surfaces are analogous to the standard hyperboloids $t^{2}-x^{2}-y^{2}-z^{2}=(3 / K)^{2}$ in Minkowski space, which provide the simplest example. A wider class of important standard examples are provided by the constant mean curvature (CMC) slicings, defined as $K=$ const., which are important for cosmology and very well studied in spherical symmetry [39-41]. For 
the purpose of comparing our results to CMC slicing, it is sufficient to recall that the stationary lapse $\alpha$ for a constant $K=\bar{K}$ in spherical symmetry reads

$$
\alpha=\sqrt{\left(\frac{\bar{K} R}{3}-\frac{C}{R^{2}}\right)^{2}+1-\frac{2 M}{R},}
$$

where $R$ is the areal radius, $M$ is the mass of the Schwarzschild black hole and $C$ is an integration constant that is of no interest for us.

The key difference between a Cauchy slice and a hyperboloidal slice of an asymptotically flat spacetime, for the purpose of this paper, is in the asymptotic behavior of the standard lapse function $\alpha$ and shift vector $\beta^{a}$ [42]. It is well known that for a Cauchy foliation one has that $\alpha \rightarrow 1$ and $\left|\beta^{a}\right| \rightarrow 0$ as $r \rightarrow \infty$, while for hyperboloidal foliations we have that

$$
\alpha=\mathcal{O}(r), \quad\left|\beta^{a}\right|=\mathcal{O}\left(r^{2}\right) \quad \text { for } \quad r \rightarrow \infty,
$$

where $r$ is a radial coordinate that asymptotically behaves like the Schwarzschild radial coordinate. For a stationary hyperboloidal slice that reaches out to $\mathscr{I}^{+}$, where the mean extrinsic curvature asymptotes to a positive value, the shift vector points inward at large separation, while at $\mathscr{I}^{-}$it points outward. This is intuitively clear from looking at a Penrose diagram such as figure 11: in order to keep $\mathscr{I}^{+}$, say, at a constant coordinate location, the shift vector has to point along $\mathscr{I}^{+}$, i.e., along an ingoing null surface. Likewise, to keep a black-hole horizon in place, an outward pointing shift vector is required, and consequently the shift vector must be expected to change its sign for stationary slices that connect a black hole to $\mathscr{I}^{+}$(or a white hole to $\mathscr{I}^{-}$). Some attention to the direction of the shift vector will be required below.

\subsection{Bona-Massó slicing conditions}

In 1994, Bona et al [29] proposed a family of slicing conditions that are formulated as evolution equations for the lapse $\alpha$. Introducing a positive, otherwise arbitrary function $f(\alpha)$, these conditions can be written as

$$
\left(\partial_{t}-\mathcal{L}_{\beta}\right) \alpha=\alpha^{2} f(\alpha)\left(K-K_{0}\right),
$$

where $\mathcal{L}_{\beta}$ denotes the Lie derivative along the shift vector $\beta^{a}$. For $f=1$ we recover harmonic slicing, $f=n / \alpha, n \in \mathbb{R}$ is called $1+\log$ slicing (with $n=2$ the most popular choice for black hole evolutions); maximal slicing can be viewed as corresponding to the limit $f \rightarrow \infty$ (see, e.g., our work [37]). The general properties of these slicing conditions have been studied in much detail [29, 35, 37, 43-47] and they have been implemented in numerical simulations with great success, in particular in the breakthrough simulations that have established the moving puncture method [6,7]. So far these conditions have been used in the context of asymptotically Euclidean foliations, and the function $K_{0}$ has usually been taken as a constant, and explicitly or implicitly been set to zero.

In this paper, we ask the question of whether the slicing condition (3) is also useful in the hyperboloidal context. We will still consider $K_{0}$ a constant for simplicity, but we will now take it seriously. We find that the value of $K_{0}$ indeed has a strong influence on the stationary foliations of the Schwarzschild spacetime, which for $K_{0} \neq 0$ differ considerably from the stationary solutions found in $[35,37,39,47]$.

Note first that for $K_{0}=0$ stationary solutions will satisfy

$$
\mathcal{L}_{\beta} \alpha=-\alpha^{2} f(\alpha) K,
$$

which is indeed consistent with $\alpha \rightarrow 1, \beta^{a} \rightarrow 0, K \rightarrow 0$ at infinity, i.e., the condition is consistent with asymptotically Euclidean slices. Looking at the right-hand side (RHS) of (3), 
one finds that $K_{0}>0$ locally has a decreasing effect on the lapse, while $K_{0}<0$ has the effect of increasing the lapse function. The question arises whether $K_{0} \neq 0$ is compatible with stationary slices of the Cauchy type. At first sight one might naively guess that $K_{0}>0$ leads to asymptotically constant-mean-curvature (CMC) slicings that reach $\mathscr{I}^{+}$, whereas $K_{0}<0$ leads to asymptotically CMC slicings that reach $\mathscr{I}^{-}$. We will see that this is indeed the case, when regular CMC slices exist. For an asymptotically Euclidean slice, however, the RHS of (3) approaches a constant if $K_{0} \neq 0$, while the left-hand side must go to zero, and therefore a stationary asymptotically Euclidean slice is incompatible with the choice of $K_{0} \neq 0$. We will now investigate the construction of asymptotically CMC slices.

\section{Construction of stationary hyperboloidal solutions}

\subsection{1+log Slicing}

We start our detailed analysis with the general version of $1+\log$ slicing and $n=2$,

$$
\left(\partial_{t}-\mathcal{L}_{\beta}\right) \alpha=2 \alpha\left(K-K_{0}\right) .
$$

After introducing our notation, we shall show explicitly that for $K_{0} \neq 0$ there are no overall regular time-independent solutions of (5) in spherical symmetry that lead to a Cauchy foliation or become asymptotically CMC slices.

The calculations are restricted to the spherically symmetric spacetime of a Schwarzschild black hole with total mass $M$,

$$
\mathrm{d} s^{2}=-\left(1-\frac{2 M}{R}\right) \mathrm{d} T^{2}+\left(1-\frac{2 M}{R}\right)^{-1} \mathrm{~d} R^{2}+R^{2} \mathrm{~d} \Omega^{2},
$$

where $T$ and $R$ denote the standard Schwarzschild coordinates and $\mathrm{d} \Omega=\mathrm{d} \phi^{2}+\sin ^{2} \theta \mathrm{d} \theta^{2}$. In the case of stationary (i.e., time-independent) foliations, the trace of the extrinsic curvature can be expressed as [39]

$$
K=-\beta^{\prime}-\frac{2 \beta}{R}
$$

where ' denotes the derivative with respect to the areal radius $R$ and

$$
\beta=\beta^{R} / \alpha= \pm \sqrt{\beta^{i} \beta_{i}} .
$$

Furthermore, the lapse and the shift are related through

$$
\alpha^{2}-\beta^{2}=1-\frac{2 M}{R}
$$

Thus, time-independent solutions of (5) satisfy

$$
\begin{aligned}
& \beta \alpha^{\prime}=-2\left(K-K_{0}\right)=2 \beta^{\prime}+\frac{4 \beta}{R}+2 K_{0} \\
& \Rightarrow \quad \alpha^{\prime}=\frac{4 R \alpha^{2}+6 M+2 R\left(K_{0} R \sqrt{\alpha^{2}+\frac{2 M}{R}-1}-2\right)}{R\left(R \alpha^{2}-2 R \alpha+2 M-R\right)} .
\end{aligned}
$$

Note that $\beta$ was defined as the positive root of (9) to obtain (11). We could as well have chosen the negative sign, which has the same effect in (11) as changing the sign of $K_{0}$. For clarity, we make the following statements for the class of solutions that have positive shift for small $R$. However, it should be kept in mind that swapping the sign both of $\beta$ and $K_{0}$ has no effect on the solution for the lapse. In Appendix A we also write a coupled system of differential 
equations for lapse and shift, which is equivalent to (9), (11) and where an explicit tracking of the sign of the shift is not necessary.

By closely investigating (10) we immediately convince ourselves that the stationary lapse can neither go to unity, nor can it be CMC-like in the range of large $R$. For $\alpha \rightarrow 1$, we find that $\alpha^{\prime}, \beta$ and $\beta^{\prime}$ vanish in the limit $R \rightarrow \infty$, which contradicts (10) for all $K_{0} \neq 0$. If $\alpha \simeq R$ and $\alpha^{\prime} \rightarrow$ const., as in the case of the stationary CMC solution (1), we can conclude $\beta \alpha^{\prime} \simeq R$, whereas the RHS of (10) approaches a constant. The only power law that does not lead to a contradiction is

$$
\alpha \simeq \sqrt{R} \Rightarrow K \simeq 1 / \sqrt{R}
$$

and below we will indeed find a family of solutions that show this asymptotic behavior for large values of $R$ and positive $K_{0}$.

However, let us first discuss solutions with negative $K_{0}$ (and positive shift). We analyze the RHS of (11) by noting that the denominator vanishes along the curve

$$
\frac{R}{M}=\frac{2}{1+2 \alpha-\alpha^{2}},
$$

that is characterized by the limit $\alpha \rightarrow 1+\sqrt{2}$ for $R \rightarrow \infty$. The numerator of (11) vanishes for

$$
\alpha=\sqrt{1-\frac{3 M}{2 R}+\frac{K_{0}^{2} R^{2}}{8}-\frac{K_{0} \sqrt{R}}{8} \sqrt{K_{0}^{2} R^{3}+8 M}},
$$

which grows unboundedly for $R \rightarrow \infty$ and $K_{0}<0$. For every point $(\alpha, R)$, we can easily determine the sign of $\alpha^{\prime}$ by investigating in which region it is located between the functions (13) and (14). If a curve that solves the differential equation (11) enters a region with negative slope, it is already clear from figure 1 that it will be driven towards the dashed curve (13), either directly in region (IV) or by going from (I) to (II). Note that from the discussion above we know that $\alpha^{\prime}$ cannot vanish in the limit $R \rightarrow \infty$. One might think that solutions can 'escape' to infinity in region (III), but for sufficiently large $\alpha$ and $R$, equation (11) reveals

$$
\alpha^{\prime} \leqslant \text { const. }\left(\frac{4}{R}+\frac{2 K_{0}}{\alpha}\right)
$$

so that for further increasing $R$ and $\alpha$, all solutions coming from (III) must enter (IV) by crossing the line (14).

This finally leads us to the conclusion that every integral curve which satisfies (11) will inevitably hit the function (13) and become singular. Put another way, for negative $K_{0}$ there exist no smooth solutions of (11) that are well defined for arbitrarily large $R$. For completeness, we note that the picture changes for $K_{0}<-2.5896 / M$, since then the functions (13) and (14) do not intersect at positive $R$ and $\alpha$. However, our arguments are based on the limit $R \rightarrow \infty$ and therefore still hold for this case.

Let us proceed to positive $K_{0}$. It is easy to prove that now the curve of vanishing numerator (14) approaches the limit $\alpha \rightarrow 1$ for $R \rightarrow \infty$. Thus, there exist solutions of (11) that do not become singular but are well defined for arbitrarily large $R$ (and $\alpha>1+\sqrt{2}$ ). We confirmed numerically that these are the predicted solutions with $\alpha \simeq 2 \sqrt{K_{0} R}(R \rightarrow \infty)$. Since $\beta$ does not change its sign, the slices are characterized by a negative $K$ and thus connect the black hole to $\mathscr{I}^{-}$. A visualization of our results is given by figure 2 . One can also observe that several integral curves which cannot grow unboundedly, hit the boundary of the domain, $\alpha=\sqrt{1-2 M / R}$. At this point, we find $\beta=0$, so that (10) is no longer a well-formulated differential equation. However, by exploiting relation (9), we find

$$
\beta^{\prime}=\frac{1}{\beta}\left(\alpha \alpha^{\prime}-\frac{M}{R^{2}}\right) \rightarrow-K_{0}
$$




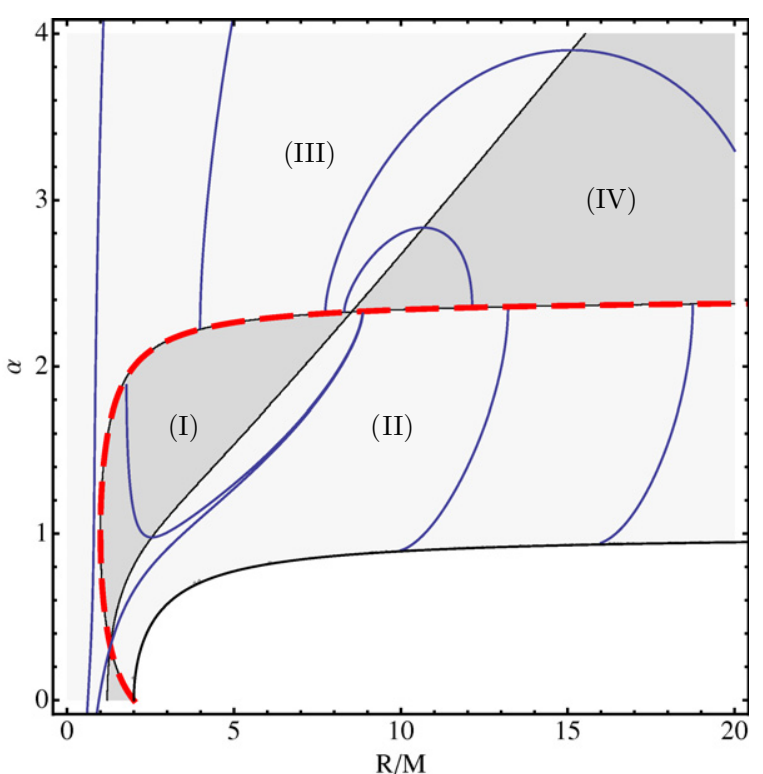

Figure 1. The analysis of stationary states that satisfy the $1+\log$ equation (5) with negative $K_{0}$ (here, $\left.K_{0}=-0.5 / M\right) . \alpha^{\prime}>0$ is indicated by regions (II) and (III), the dark gray regions (I) and (IV) illustrate $\alpha^{\prime}<0$. Their boundaries are given by (13) (dashed curve), (14) and $\alpha=\sqrt{1-2 M / R}$. All solutions become singular where the dashed curve is hit. Some examples which we have calculated explicitly are shown for clarity.

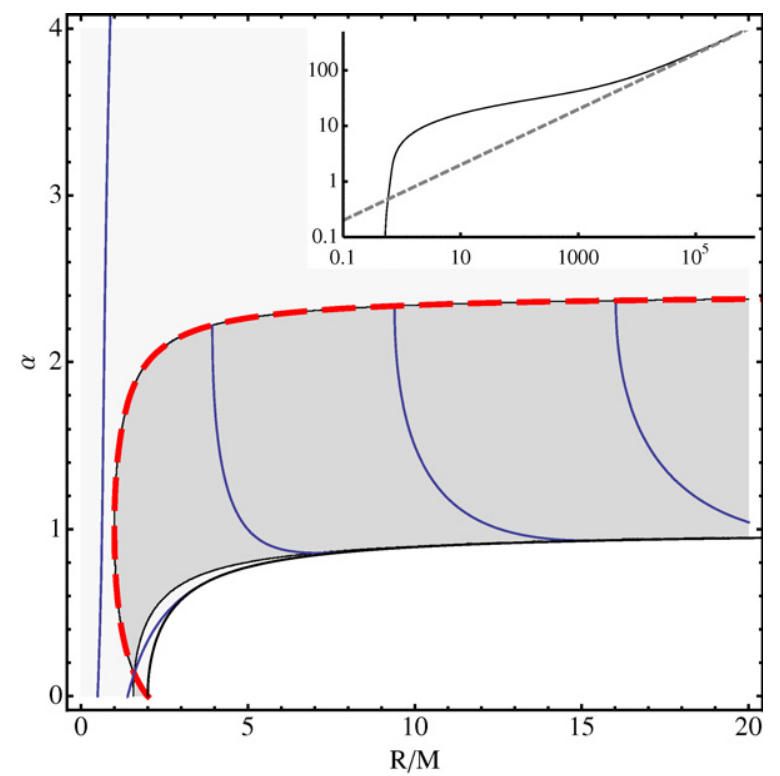

Figure 2. The equivalent of figure 1, but with positive $K_{0}$ (the chosen example is $K_{0}=0.1 / M$ ). Note that for increasing $R$, the solutions of (11) are driven away from the dashed curve, to eventually escape to infinity or change the sign of $\beta$ (see text). The included log-log plot illustrates how the outer left curve approaches $\alpha=2 \sqrt{K_{0} R}$ (straight dashed line) for large $R$. 


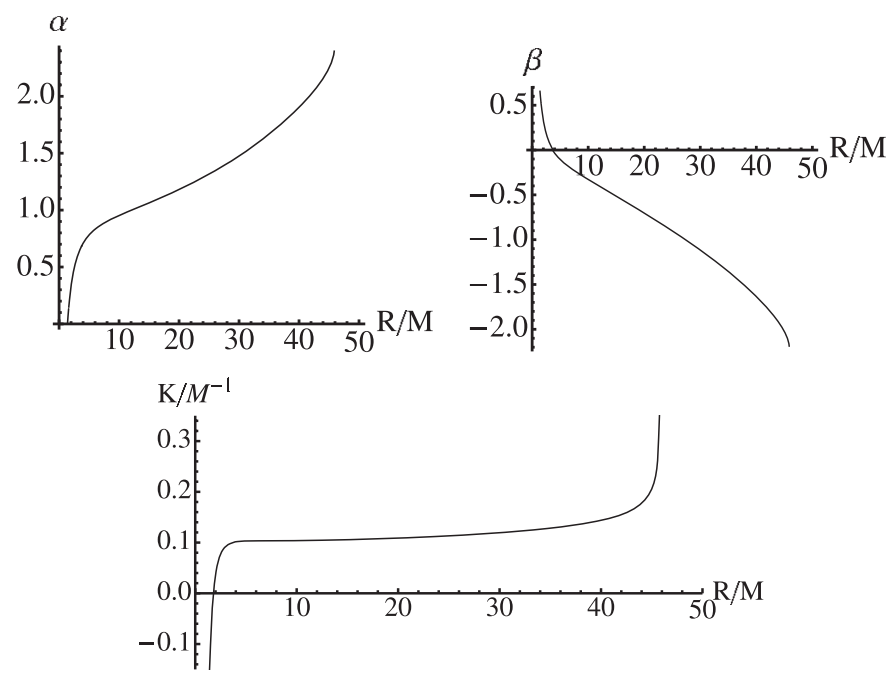

Figure 3. The lapse, the shift and the trace of the extrinsic curvature of the numerically calculated critical stationary solution of $1+\log$ slicing with offset $K_{0}=0.1 / M$. For small $R$ we recover a behavior similar to the stationary trumpet solution of standard $1+\log$ [37]. At $R \approx 3.76 M$, however, $\beta$ changes its sign and $K$ seems to settle close to $K_{0}$, before finally all quantities become singular at $R \approx 46 M$.

for $\alpha \rightarrow \sqrt{1-2 M / R}$. Hence, the shift changes its sign and becomes negative. The curves that hit the boundary of the domain can therefore be smoothly extended by allowing $\beta$ to cross zero at this point. For negative shift, (11) has to be changed as if $K_{0} \mapsto-K_{0}$. We therefore conclude that all these solutions run into a singularity at finite $R$. Interestingly, the unique solution that is defined as the one that crosses the common root of (13) and (14) (see for example [37, 48, 49] for the discussion of the standard 1+log case) is of this type. Figure 3 shows this particular example, which we refer to as the 'critical solution'. As we see, due to the discussed properties of (11) there is no overall regular critical stationary solution of $1+\log$ slicing with offset $K_{0} \neq 0$.

\subsection{Harmonic slicing}

In this section, we study the harmonic slicing condition

$$
\left(\partial_{t}-\mathcal{L}_{\beta}\right) \alpha=\alpha^{2}\left(K-K_{0}\right) .
$$

We find that in this case, $K_{0} \neq 0$ does lead asymptotically to CMC solutions at large distances from the black hole. As expected, care has to be taken in assuming the sign of the shift, since for positive $K_{0}$ it changes from ' + ' inside the black hole to ' - ' in the limit $R \rightarrow \infty$.

The analysis of harmonic slicing can be motivated by considering the generalized BonaMassó slicing condition (3), restricted to $f(\alpha)=\alpha^{\kappa}$. First, $\kappa$ may be any exponent. Looking for stationary solutions that may asymptotically approach the CMC solution (1), we exploit the assumption $\alpha \simeq|\bar{K}| R / 3(R \rightarrow \infty)$ to determine $\kappa$ and $\bar{K}$. Starting from (3), we find

$$
\begin{aligned}
\beta \alpha^{\prime} & =-\alpha^{\kappa+1}\left(K-K_{0}\right) \\
& \Rightarrow \frac{|\bar{K}| \bar{K} R}{9} \simeq-\left(K_{0}-\bar{K}\right)\left(\frac{|\bar{K}| R}{3}\right)^{\kappa+1} .
\end{aligned}
$$



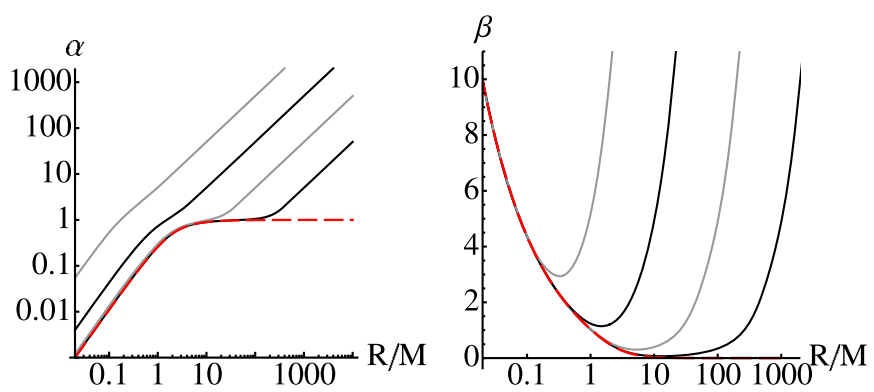

Figure 4. The stationary lapse (left) and shift (right) of harmonic slicing (17). Each curve is obtained by the unique solution of (21) that passes the regular singular point (22). The following cases are displayed (from top to bottom) $M K_{0}=-10,-1,-0.1,-0.01$, 0 , where vanishing $K_{0}$ is shown as a dashed line.

By comparing the exponent of $R$ on both sides of the estimate, we conclude that $\kappa=0$ and

$$
\bar{K} \rightarrow \frac{3 K_{0}}{2} \quad(R \rightarrow \infty)
$$

Vanishing $\kappa$ obviously leads to the slicing condition (17).

We now discuss time-independent solutions of (17) in analogy with our analysis of the $1+\log$ condition in the previous section. We note that stationary states with positive shift satisfy equivalently to (17) the following ordinary differential equation,

$$
\alpha^{\prime}=\frac{\alpha\left[3 M+R\left(2 \alpha^{2}-2+K_{0} R \sqrt{\alpha^{2}+\frac{2 M}{R}-1}\right)\right]}{(2 M-R) R} .
$$

The set of points of vanishing numerator for $R>0, \alpha>0$ is still described by (14). The denominator is zero at the horizon, $R=2 M$. In this special case, we can analytically express the regular singular point $\left(R_{s}, \alpha_{s}\right)$, at which both functions intersect, as

$$
\alpha_{s}=\frac{1}{2} \sqrt{1+2 K_{0}^{2}-2 K_{0} \sqrt{K_{0}^{2}+1}}, \quad R_{s}=2 M .
$$

Note that we face a different situation than in the $1+\log$ case. Here, the curve of vanishing denominator is a vertical line in the $\alpha-R$ diagram. Therefore, every solution of (21) that smoothly connects the inside and the outside of the black hole must cross the line of vanishing denominator. To prevent the integral curve to become singular there, the numerator should vanish at the same point, i.e., the unique regular stationary lapse must pass the point given by (22).

We analyze the resulting critical solution for the lapse by numerically integrating (21). Our results show that $K$ indeed asymptotically approaches $3 K_{0} / 2$ for large $R$ in the case of both negative and positive $K_{0}$. The plots for different choices of $K_{0}$ are shown in figures 5 and 7. Furthermore, the left panels of figures 4 and 6 confirm that the lapse asymptotically grows unboundedly similar to CMC slicing, i.e., $\alpha \simeq|K| R / 3 \simeq\left|K_{0}\right| R / 2$.

For negative $K_{0}$, the shift $\beta$ is decreasing for small $R$ until the $K_{0}$-dependent minimum is reached, then the slope changes its sign and $\beta \rightarrow \infty$ for $R \rightarrow \infty$. Thus, the shift is positive everywhere. The trace of the extrinsic curvature remains negative for all $R$. We conclude that the stationary foliation of harmonic slicing with negative offset connects the singularity $R=0$ (at infinite proper distance) to past lightlike infinity $\mathscr{I}^{-}$. 


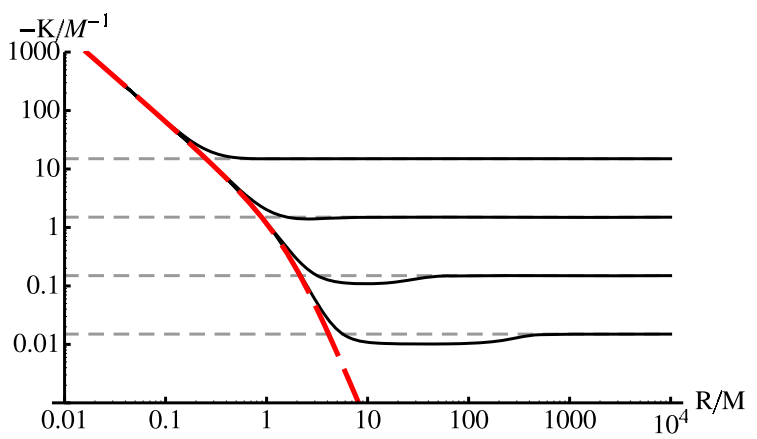

Figure 5. The (negative) trace of the extrinsic curvature of the stationary states shown in figure 4 . The horizontal dashed lines correspond to $3 K_{0} / 2$, the long-dashed curve illustrates the analytically known case of vanishing $K_{0}$.
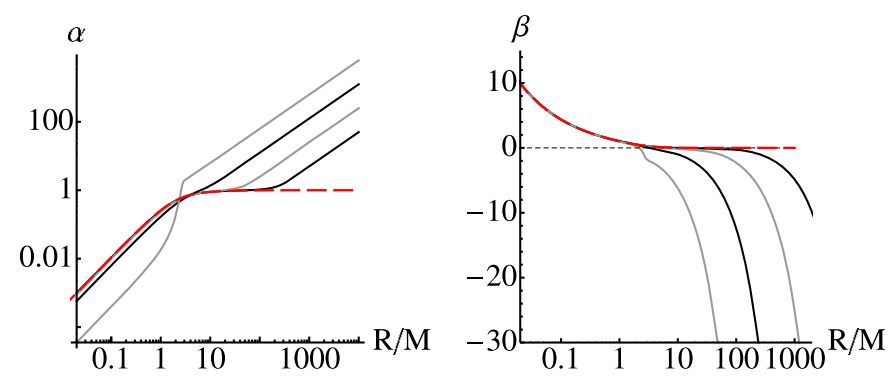

Figure 6. The stationary lapse (left) and shift (right) of harmonic slicing (17) with non-negative offset $M K_{0}=1.25,0.25,0.05,0.01,0$ (from top to bottom on the left panel, vice versa on the right panel; $K_{0}=0$ as a dashed line).

Positive offset, on the other hand, always leads to stationary states where $\alpha$ hits the boundary of the domain, $\alpha=\sqrt{1-2 M / R}$. $\beta$ vanishes at these points and changes its sign from positive to negative, as described for $1+\log$ in the previous section. By similar calculations one easily proves that again

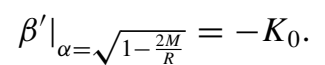

The numerical calculations also suggest that the root of the shift converges monotonically to the horizon for increasing $K_{0}$, which may lead to problems when numerically solving (21) for large $K_{0}$. However, as a proof of principle we show several choices $K_{0} \leqslant 1.25 M^{-1}$ in figures 6 and 7. The important difference to the solutions with negative offset is that here, changing the sign of $\beta$ induces a change of the sign of $K$. Asymptotically approaching a constant positive $K$ yields a slicing that smoothly connects the singularity of the black hole with future null infinity $\mathscr{I}^{+}$.

\subsection{Combined approach}

Let us summarize what we have found in the previous sections. Starting from a general form of Bona-Massó slicing (3), we analyzed the $1+\log$ case $(f=2 / \alpha)$. After concluding that in this case there is no overall regular stationary state that connects the black hole to $\mathscr{I}^{+}$, we found 


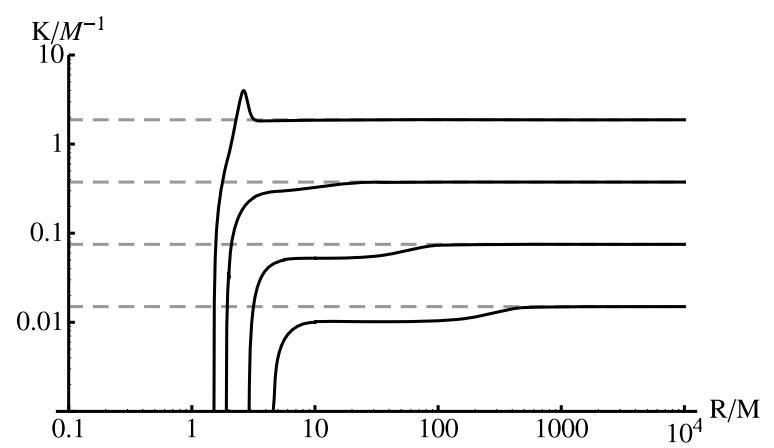

Figure 7. The trace of the extrinsic curvature of the stationary solutions that are displayed in figure 6. The dashed lines correspond to $3 K_{0} / 2$.

that harmonic slicing $(f=1)$ is suitable for having asymptotically CMC-like stationary states. The drawback in this case is that harmonic slicing is only marginally singularity avoiding the slicing comes arbitrarily close to the singularity of the black hole. What we propose now is to combine both attractive properties, the strong singularity avoidance of $1+\log$ and the asymptotically constant $K>0$ of harmonic slicing (with positive offset) by simply adding both conditions in an appropriate way,

$$
\begin{aligned}
f & =\frac{2}{\alpha}+1 \\
& \Rightarrow\left(\partial_{t}-\mathcal{L}_{\beta}\right) \alpha=\left(2 \alpha+\alpha^{2}\right)\left(K-K_{0}\right) .
\end{aligned}
$$

The function $f$ is obviously dominated by the $1+\log$ part for small $\alpha$ (i.e., inside the black hole) and by the harmonic part for large $\alpha$ (which is in the case of CMC slicing equivalent to large distances from the black hole).

We analyze stationary solutions of (25) in the case of a Schwarzschild spacetime by using the established methods and equations (recall especially (7), (8) and (9)). The timeindependent form of (25) can be written as

$$
\alpha^{\prime}=\frac{(2+\alpha)\left[3 M+R\left(2 \alpha^{2}-2+K_{0} R \sqrt{\alpha^{2}+\frac{2 M}{R}-1}\right)\right]}{R[2 M-R(1+2 \alpha)]} .
$$

The critical solution we consider is defined by passing the common root of the numerator (see (14) that is valid for arbitrary $f$ ) and denominator

$$
\frac{R}{M}=\frac{2}{1+2 \alpha} \quad \Leftrightarrow \quad \alpha=\frac{2 M-R}{2 R} .
$$

Before we further investigate the set of solutions for positive $K_{0}$, we give some exact analytical expressions for the case $K_{0}=0$. Under this assumption, time-independent solutions of (25) satisfy

$$
\begin{aligned}
& \beta \alpha^{\prime}=(2+\alpha)\left(\beta^{\prime}+\frac{2 \beta}{R}\right) \\
& \Leftrightarrow \quad \int \frac{\mathrm{d} \alpha}{2+\alpha}=\int\left(\frac{\beta^{\prime}}{\beta}+\frac{2}{R}\right) \mathrm{d} R \\
& \Leftrightarrow \quad \alpha^{2}=1-\frac{2 M}{R}+\frac{C^{2}(2+\alpha)^{2}}{R^{4}} .
\end{aligned}
$$




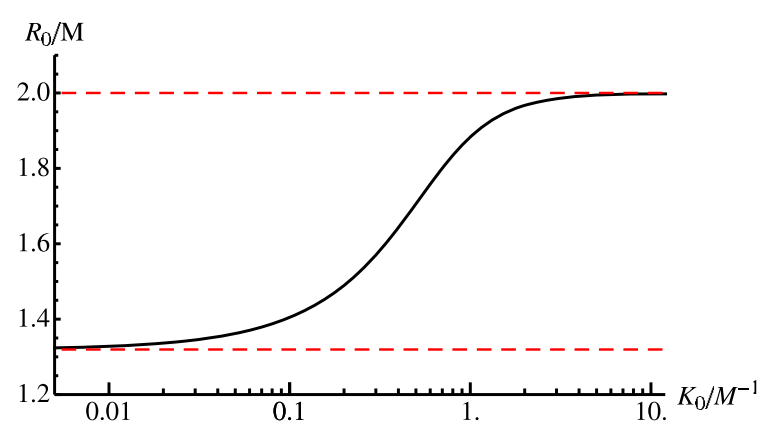

Figure 8. The throat (root of the lapse) of the critical stationary solutions of (25). The horizontal dashed lines correspond to the limits $R_{0} \rightarrow 2 M$ for $K_{0} \rightarrow \infty$ and $R \rightarrow 1.3955 M$ (see the text) for $K_{0} \rightarrow 0$.

The (positive) integration constant $C^{2}$ is determined by picking the critical solution as described above. As the regular singular point, we find

$$
\begin{aligned}
& R_{s}=\frac{\sqrt{13}+1}{3} M \approx 1.535 M, \\
& \alpha_{s}=\frac{\sqrt{13}-3}{4} \approx 0.151,
\end{aligned}
$$

and the constant is given by

$$
C^{2}=\frac{8}{243}(13 \sqrt{13}-35) M^{4} \approx 0.391 M^{4} .
$$

The implicit solution (28) is now uniquely determined and can also be written explicitly as roots $R(\alpha)$. Note that the roots have to be picked carefully in order to obtain the smooth, monotonically increasing function we are interested in (see also [49] for a similar discussion). One important feature is that the resulting lapse starts at $R_{0}>0$ with $\alpha\left(R_{0}\right)=0$ and $\alpha^{\prime}\left(R_{0}\right)>0$. Hence, the behavior of strongly singularity-avoiding conditions is retained and, expressed in the terminology of [37], the region close to the black hole is a 'trumpet' with the throat $R_{0} \approx 1.3955 M$. However, the case $K_{0}=0$ reveals $\alpha \rightarrow 1$ for $R \rightarrow \infty$, and is therefore not qualitatively different from the standard $1+\log$.

If we now allow $K_{0}$ to be positive, we find by numerically integrating (26) that $\alpha$ still starts at the throat $R_{0}\left(K_{0}\right)$ (see figure 8) but then follows the behavior that was already observed for harmonic slicing, which means that, at finite $R$, the first positive shift changes its sign and the lapse asymptotically approaches the CMC limit $\alpha \simeq K_{0} R / 2$. The trace of the extrinsic curvature changes sign as well (from negative to positive) and approaches the predicted limit $3 K_{0} / 2$ for $R \rightarrow \infty$. Our results are displayed in figures $8-10$.

In order to point out the new quality of the proposed slicing, we also calculate the embedding in a Carter-Penrose diagram. For this purpose, we transform our integrated quantities $\alpha(R)$ and $\beta(R)$ to standard Schwarzschild coordinates using the height function approach $[39,50]$. Transforming these coordinates to Kruskal coordinates and compactifying them is straightforward and was already described in [37]. The resulting diagram is shown in figure 11. As discussed before, the slices indeed go towards $\mathscr{I}^{+}$(recall, positive $K$ ) and approach timelike infinity $i_{L}^{+}$along the throat, as it is typical for strongly singularity-avoiding slices. 

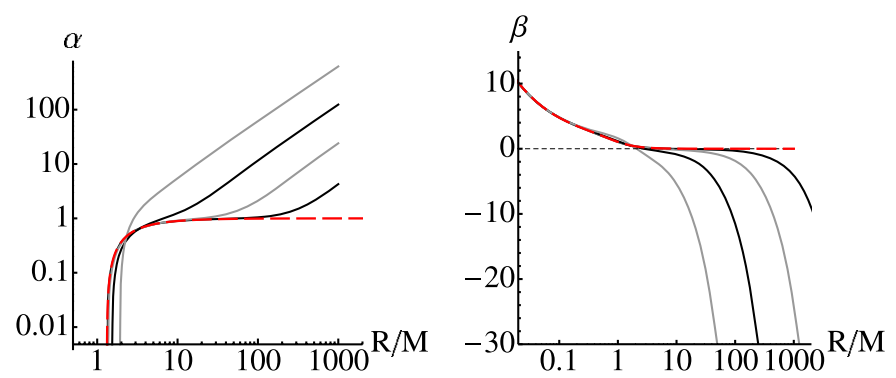

Figure 9. The stationary lapse (left) and shift (right) of our combined slicing condition (25) with non-negative offset $M K_{0}=1.25,0.25,0.05,0.01,0$ (from top to bottom on the left panel, vice versa on the right panel; $K_{0}=0$ as a dashed line).

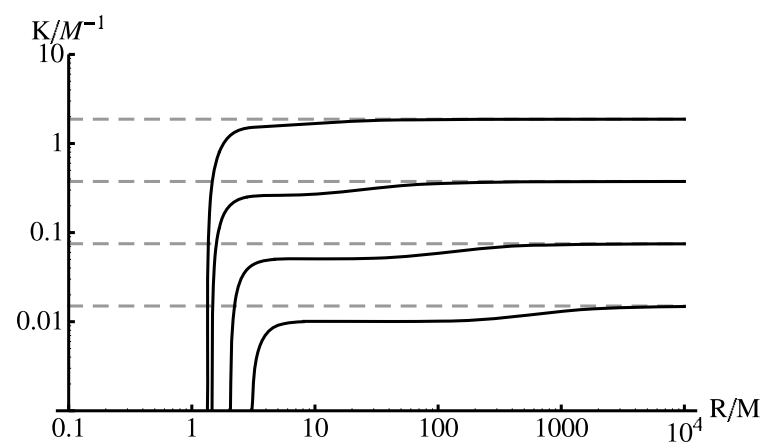

Figure 10. The trace of the extrinsic curvature of the stationary solutions that are displayed in figure 9. The dashed lines correspond to $3 K_{0} / 2$.

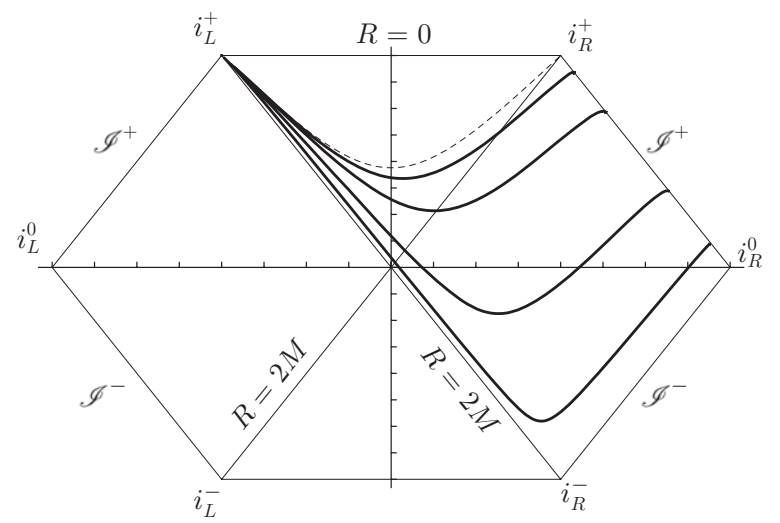

Figure 11. The Carter-Penrose diagram of the slicing defined by the critical stationary solution of (25) and $K_{0}=1 M^{-1}$. The dashed line illustrates the curve of constant $R=R_{0} \approx 1.8831 M$. All slices connect the throat $R_{0}$ to $\mathscr{I}^{+}$. Each displayed time step is $5 M$. 


\section{Discussion}

Let us summarize our results.

For the $1+\log$ slicing condition used in moving-puncture simulations of black-hole spacetimes, we have previously found that it is possible to find a stationary solution for a Schwarzschild black hole. This solution represents a trumpet geometry: the slice extends from a throat at some finite value $R_{0}$ of the Schwarzschild radial coordinate out to spatial infinity [35-37]. In the present paper we have extended these results to spacelike slices that instead reach null infinity, and which are commonly referred to as hyperboloidal slices. Such hyperboloidal slices asymptotically approach a finite value of the mean extrinsic curvature, and include the constant-mean-curvature slices. One potential approach to produce trumpet slices that also extend to null infinity is to modify the standard $1+\log$ slicing condition to include an offset term that leads to constant-mean-curvature at large distances. We have shown that there are no regular slices that satisfy this condition, given by (5). Also, such an offset term is an obstruction to construct regular stationary Cauchy slices for the $1+\log$ slicing condition.

If we instead deal with the analogous modification of harmonic slicing, equation (17), regular stationary slices do exist. However, harmonic slices reach the black-hole singularity, and for moving-puncture-like simulations we would prefer singularity-avoiding slices. Both requirements - singularity-avoiding $1+\log$ slicing near the black hole and CMC harmonic slices at null infinity - can be met by using a hybrid slicing condition of the form suggested in $(25)$,

$$
\left(\partial_{t}-\mathcal{L}_{\beta}\right) \alpha=\left(2 \alpha+\alpha^{2}\right)\left(K-K_{0}\right) .
$$

We have demonstrated the efficacy of this approach by constructing slices of the Schwarzschild spacetime that extend from $R_{0}\left(1.3955 M<R_{0}<2 M\right.$, dependent on the choice of $\left.K_{0}\right)$ to null infinity. Both for the hybrid condition and harmonic slicing, the sign of the trace of the extrinsic curvature (and therefore the offset $\left.K_{0}\right)$ is directly related to the outer end $(R \rightarrow \infty)$ of the slices via

$$
K \rightarrow \frac{3}{2} K_{0} \Rightarrow \text { slices extend to }\left\{\begin{array}{lll}
\mathscr{I}^{+} & \text {for } & K_{0}>0 \\
\mathscr{I}^{-} & \text {for } & K_{0}<0 .
\end{array}\right.
$$

There is obviously a large freedom in constructing gauge conditions and stationary slicings with similar properties, and in choosing (or generalizing to a function) the constant $K_{0}$. What works best will presumably depend to some degree on the application.

The results presented in this paper are intended as first steps toward producing multipleblack-hole 'hyperboloidal trumpet' data. Numerically evolving such data would require a sufficiently regular treatment of null infinity in Einstein's equations. The philosophy we advocated in the introduction was that the most practical approach may be to perform the regularization of Einstein's equations after choosing the type of initial data to be evolved, and the gauge conditions to be employed. As such, we suggest the data and gauge conditions we have presented here as one possible starting point for deriving a method for null evolutions that is also close in spirit to current moving-puncture simulations.

\section{Acknowledgments}

We thank Anıl Zenginoğlu, Bernd Brügmann and Lucía Santamaría for valuable comments on our manuscript. Frank Ohme and Mark Hannam thank the University of the Balearic Islands, and Sascha Husa thanks University College Cork for hospitality, while some of this work was carried out. Mark Hannam and Niall Ó Murchadha were supported by SFI grant 07/RFP/PHYF148. This work was supported in part by the DFG grant SFB/Transregio 
7 'Gravitational Wave Astronomy'. S Husa has been supported in part as a VESF fellow of the European Gravitational Observatory (EGO), by DAAD grant D/07/13385 and grant FPA-2007-60220 from the Spanish Ministerio de Educación y Ciencia.

\section{Appendix. Bona-Massó slicing stationary equations as a coupled system for lapse and shift}

In the main text we have used a single differential equation for the lapse and the quadratic algebraic equation (9) to construct our slicings-tracking the solution of (9) with the appropriate sign of the shift vector. For completeness, we now also write a coupled system of differential equations for lapse and shift, where an explicit tracking of the sign of the shift is not necessary. We write the metric of a Schwarzschild black hole as

$$
\mathrm{d} s^{2}=-\left(\alpha^{2}-\gamma\left(\beta^{R}\right)^{2}\right) \mathrm{d} t^{2}+2 \gamma \beta^{R} \mathrm{~d} t \mathrm{~d} R+\gamma \mathrm{d} R^{2}+R^{2} \mathrm{~d} \Omega^{2},
$$

where $t$ is the time coordinate of the stationary slice, $R$ is the standard Schwarzschild radial coordinate, $\mathrm{d} \Omega=\mathrm{d} \phi^{2}+\sin ^{2} \theta \mathrm{d} \theta^{2}$, and $\alpha, \beta_{R}$ and $\gamma$ are functions of $R$. One finds from the Einstein equations that $\gamma=1 / \alpha^{2}$. Note that we now use the radial shift component $\beta^{R}$ instead of $\beta$, in order to keep track of the direction of the shift. In the case of time-independent foliations the extrinsic curvature reads

$$
K=-\frac{\left(r^{4}\left(\beta^{R}\right)^{2} \gamma\right)^{\prime}}{2 r^{4} \alpha \beta^{R} \gamma}
$$

where again ' denotes the derivative with respect to the areal radius $R$. The Einstein equations together with the stationary Bona-Massó slicing condition (3) then imply

$$
\alpha^{\prime}=\frac{f(\alpha)\left(K_{0} r^{2} \beta^{R}+2 r \alpha^{3}+(3-2 r) \alpha\right)}{r \alpha(r \alpha(\alpha-f(\alpha))-r+2)},
$$

and

$\beta^{R^{\prime}}=\frac{f(\alpha)\left(2 K_{0} r \alpha^{3}\left(2 r \alpha^{2}-r+2\right)+\alpha^{2} \beta^{R}\left(7 r \alpha^{2}-3 r+4\right)+r\left(\beta^{R}\right)^{3}\right)-2 \alpha^{3} \beta^{R}}{2 r \alpha^{3}(r \alpha(\alpha-f(\alpha))-r+2)}$,

where $r=R / M$.

\section{References}

[1] Hannam M et al 2009 The Samurai project: verifying the consistency of black-hole-binary waveforms for gravitational-wave detection Phys. Rev. D 79084025

[2] Danzmann K et al 1998 Lisa pre-phase a report Max-Planck-Inistitut für Quantenoptik, Rep. MPQ 233 184-209

[3] Danzmann K and Rudiger A 2003 LISA technology—concept, status, prospects Class. Quantum Grav. 20 S1-9

[4] Einstein Telescope http://www.et-gw.eu/, a Design Study project supported by the European Commission under the Framework Programme 7 (FP7, Grant Agreement 211743)

[5] Pretorius F 2005 Evolution of binary black hole spacetimes Phys. Rev. Lett. 95121101

[6] Campanelli M, Lousto C O, Marronetti P and Zlochower Y 2006 Accurate evolutions of orbiting black-hole binaries without excision Phys. Rev. Lett. 96111101

[7] Baker J G, Centrella J, Choi D-Il, Koppitz M and van Meter J 2006 Gravitational wave extraction from an inspiraling configuration of merging black holes Phys. Rev. Lett. 96111102

[8] Pretorius F 2007 Binary black hole coalescence Physics of Relativistic Objects in Compact Binaries: From Birth to Coalescence (Astrophysics and Space Science Library vol 359) ed M Colpi, P Casella, V Gorini, U Moschella and A Possenti (Netherlands: Springer) pp 305-69

[9] Hannam M 2009 Status of black-hole-binary simulations for gravitational-wave detection Class. Quantum Grav. 26114001

[10] Penrose R 1963 Asymptotic properties of fields and space-times Phys. Rev. Lett. 10 66-8 
[11] Frauendiener J 2000 Numerical treatment of the hyperboloidal initial value problem for the vacuum Einstein equations: III. On the determination of radiation Class. Quantum Grav. 17 373-87

[12] Purrer M, Husa S and Aichelburg P C 2005 News from critical collapse: Bondi mass, tails and quasinormal modes Phys. Rev. D 71104005

[13] Babiuc M, Szilagyi B, Hawke I and Zlochower Y 2005 Gravitational wave extraction based on Cauchycharacteristic extraction and characteristic evolution Class. Quantum Grav. 22 5089-108

[14] Babiuc M C, Bishop N T, Szilagyi B and Winicour J 2009 Strategies for the characteristic extraction of gravitational waveforms Phys. Rev. D 79084011

[15] Winicour J 2009 Characteristic evolution and matching Living Rev. Rel. 123 (http://www.livingreviews. org/lrr-2009-3)

[16] Zenginoğlu A 2008 Hyperboloidal evolution with the Einstein equations Class. Quantum Grav. 25195025

[17] Zenginoğlu A, Nunez D and Husa S 2009 Gravitational perturbations of Schwarzschild spacetime at null infinity and the hyperboloidal initial value problem Class. Quantum Grav. 26035009

[18] Friedrich H 1983 Cauchy problems for the conformal vacuum field equations in general relativity Commun. Math. Phys. 91 445-72

[19] Friedrich H 2002 Conformal Einstein evolution Lect. Notes Phys. 604 1-50

[20] Frauendiener J 2002 Some aspects of the numerical treatment of the conformal field equations Lect. Notes Phys. 604 261-82

[21] Frauendiener J 2004 Conformal infinity Living Rev. Rel. 7 (http://www.livingreviews.org/lrr-2004-1)

[22] Husa S 2002 Problems and successes in the numerical approach to the conformal field equations Lect. Notes Phys. 604 239-60

[23] Husa S 2003 Numerical relativity with the conformal field equations Lect. Notes Phys. 617 159-92

[24] Calabrese G, Gundlach C and Hilditch D 2006 Asymptotically null slices in numerical relativity: mathematical analysis and spherical wave equation tests Class. Quantum Grav. 23 4829-46

[25] Andersson L 2002 Construction of hyperboloidal initial data Lect. Notes Phys. $604183-94$

[26] Husa S, Schneemann C, Vogel T and Zenginoğlu A 2006 Hyperboloidal data and evolution AIP Conf. Proc. $841306-13$

[27] Moncrief V and Rinne O 2009 Regularity of the Einstein equations at future null infinity Class. Quantum Grav. 26125010

[28] Bishop N T, Gomez R, Lehner L, Maharaj M and Winicour J 1997 High-powered gravitational news Phys. Rev. D 56 6298-309

[29] Bona C, Massó J, Seidel E and Stela J 1995 A new formalism for numerical relativity Phys. Rev. Lett. $75600-3$

[30] Andersson L and Moncrief V 2003 Elliptic-hyperbolic systems and the Einstein equations Ann. Henri Poincare 4 1-34

[31] Bonazzola S, Gourgoulhon E, Grandclement P and Novak J 2004 A constrained scheme for Einstein equations based on Dirac gauge and spherical coordinates Phys. Rev. D 70104007

[32] Cordero-Carrion I et al 2009 An improved constrained scheme for the Einstein equations: an approach to the uniqueness issue Phys. Rev. D 79024017

[33] Zenginoğlu A and Husa S 2006 Hyperboloidal foliations with SCRI-fixing in spherical symmetry vol 2 Proc. 11th Marcel Grossman Meeting On General Relativity ed H Kleinert, R T Jantzen and R Ruffini (Singapore: World Scientific) 1624-6

[34] Zenginoğlu A 2008 Hyperboloidal foliations and scri-fixing Class. Quantum Grav. 25145002

[35] Hannam M, Husa S, Pollney D, Brügmann B and Ó Murchadha N 2007 Geometry and regularity of moving punctures Phys. Rev. Lett. 99241102

[36] Hannam M et al 2007 Where do moving punctures go? J. Phys. Conf. Ser. 66012047

[37] Hannam M, Husa S, Ohme F, Brügmann B and Ó Murchadha N 2008 Wormholes and trumpets: the Schwarzschild spacetime for the moving-puncture generation Phys. Rev. D 78064020

[38] Wald R M 1984 General Relativity (Chicago: The University of Chicago Press)

[39] Malec E and Ó Murchadha N 2003 Constant mean curvature slices in the extended Schwarzschild solution and collapse of the lapse: part I Phys. Rev. D 68124019

[40] Malec E and Ó Murchadha N 2009 The general spherically symmetric constant mean curvature foliations of the Schwarzschild solution Phys. Rev. D 80024017

[41] Gentle A P, Holz D E, Kheyfets A, Laguna P, Miller W and Shoemaker D M 2001 Constant crunch coordinates for black hole simulations Phys. Rev. D 63064024

[42] York J W 1979 Kinematics and dynamics of general relativity Sources of Gravitational Radiation ed L L Smarr (Cambridge: Cambridge University Press) pp 83-126

[43] Bona C, Massó J, Seidel E and Stela J 1997 First order hyperbolic formalism for numerical relativity Phys. Rev. D 56 3405-15 
[44] David Brown J 2008 Puncture Evolution of Schwarzschild Black Holes Phys. Rev. D 77044018

[45] Alcubierre M 1997 The appearance of coordinate shocks in hyperbolic formalisms of general relativity Phys. Rev. D 55 5981-91

[46] Alcubierre M 2003 Hyperbolic slicings of spacetime: singularity avoidance and gauge shocks Class. Quantum Grav. 20 607-24

[47] Cook G B and Scheel M A 1997 Well behaved harmonic time slices of a charged, rotating, boosted black hole Phys. Rev. D 56 4775-81

[48] Garfinkle D, Gundlach C and Hilditch D 2008 Comments on Bona-Massó type slicing conditions in long-term black hole evolutions Class. Quantum Grav. 25075007

[49] Brügmann B 2009 Schwarzschild black hole as moving puncture in isotropic coordinates Gen. Rel. Grav. (DOI:10.1007/s10714-009-0818-6)

[50] Beig R and Ó Murchadha N 1998 Late time behaviour of the maximal slicing of the Schwarzschild black hole Phys. Rev. D 57 4728-37 